to

\title{
Controlling Multicompartment Morphologies Using Solvent Conditions and Chemical Modification
}

\author{
by Tina I. Löbling, Olli Ikkala, André H. Gröschel*, Axel H. E. Müller*
}

\section{Materials and Methods}

Materials. Hexyl mercaptan (95\%, Aldrich) and THF (Aldrich p.a.) were used without further purification. Dioxane and $N, N$-dimethylacetamide (DMAc p.a.) were of analytical grade and purchased from Roth. For dialysis, acetone and isopropanol of technical grade were used. Dialysis tubes of regenerated cellulose with a molecular weight cut-off of 12,000$14,000 \mathrm{~g} / \mathrm{mol}$ were purchased from Roth, equilibrated in deionized water for $10 \mathrm{~min}$ and washed with excess dioxane before use.

Polymer Synthesis. All polymers were synthesized via living anionic polymerization in either toluene $\left(\mathrm{PS}_{192}, \mathrm{~S}_{306} \mathrm{~B}_{151} \mathrm{M}_{340}\right)$ or THF $\left(\mathrm{S}_{510} \mathrm{~B}_{258} \mathrm{M}_{260}, \mathrm{~S}_{516} \mathrm{~B}_{140} \mathrm{M}_{76}, \mathrm{~S}_{516} \mathrm{~B}_{140} \mathrm{M}_{118}\right.$, $\mathrm{PMMA}_{5700}$ ) as reported elsewhere in detail. ${ }^{1,2}$ Subscripts denote the degree of polymerization (DP) of the blocks. The polymers were characterized by combination of SEC and ${ }^{1} \mathrm{H}-\mathrm{NMR}$ as summarized in Table 1.

Preparation of micellar solutions. The SBM triblock terpolymer was first dissolved in DMAc at a concentration of $0.1 \mathrm{~g} / \mathrm{L}$ and annealed over night at $70{ }^{\circ} \mathrm{C}$ to ensure formation and equilibration of micelles with PB core and PS/PMMA corona. The solution was dialyzed against the final acetone/isopropanol mixture to induce the formation of multicompartment nanostructures. For blend-experiments with PS homopolymer, a stock solution of PS was first prepared in DMAc $(1 \mathrm{~g} / \mathrm{L})$, mixed with the SBM triblock terpolymer in desired ratios and stirred for $24 \mathrm{~h}$ prior to dialysis.

Chemical modification. For the thiol-ene reaction, $200 \mathrm{mg}$ of $\mathrm{S}_{516} \mathrm{~B}_{140} \mathrm{M}_{118}$ were first dissolved in $6 \mathrm{~mL}$ THF. After complete dissolution, a 15-fold excess (compared to the PB double bonds) of hexyl mercaptan was added. The mixture was degassed for 20 min and then irradiated for $24 \mathrm{~h}$ using a UV-lamp with a cut-off filter of $\lambda=300 \mathrm{~nm}$. Through click modification, the $\mathrm{S}_{516} \mathrm{~B}_{140} \mathrm{M}_{118}$ polymer changes to $\mathrm{S}_{516} \mathrm{H}_{140} \mathrm{M}_{118}$ and the volume ratio of $V_{\mathrm{PS}} / V_{\mathrm{PB}}=3.5$ to $V_{\mathrm{PS}} / V_{\mathrm{PH}}=2.1$. The block volumes are calculated by multiplying the molar volume of the monomeric unit ( $\mathrm{PS}=99.0 \mathrm{~cm}^{3} / \mathrm{mol} ; \mathrm{PB}=56.2 \mathrm{~cm}^{3} / \mathrm{mol} ; \mathrm{PH}=174.5 \mathrm{~cm}^{3} / \mathrm{mol}$ ) with the degree of polymerization of the respective blocks. 
Table S1: Characteristics of the used polymers including degree of polymerization (DP), molar fraction of the PMMA corona $(x)$, weight fractions of the core forming blocks $(f)$, overall molecular weight $\left(M_{n}\right)$ and dispersity $(\theta)$.

\begin{tabular}{|c|c|c|c|c|c|c|c|c|}
\hline Sample $^{a}$ & DP (S) & DP (B) & DP (M) & $x_{M}$ & $f_{\mathrm{S}}$ & $f_{\mathrm{B}}$ & $M_{\mathrm{n}}[\mathrm{kg} / \mathrm{mol}]^{b}$ & $\boldsymbol{D}^{c}$ \\
\hline $\mathrm{PS}_{192}$ & 192 & - & - & - & 1 & - & 20 & 1.02 \\
\hline PMMA $_{5700}$ & - & - & 5700 & 1 & - & - & 570 & 1.12 \\
\hline $\mathrm{S}_{306} \mathrm{~B}_{151} \mathrm{M}_{340}$ & 306 & 151 & 340 & 0.43 & 0.43 & 0.11 & 74 & 1.06 \\
\hline $\mathrm{S}_{510} \mathrm{~B}_{258} \mathrm{M}_{260}$ & 510 & 258 & 260 & 0.25 & 0.57 & 0.15 & 93 & 1.04 \\
\hline $\mathrm{S}_{516} \mathrm{~B}_{140} \mathrm{M}_{76}$ & 516 & 140 & 76 & 0.10 & 0.78 & 0.11 & 69 & 1.05 \\
\hline $\mathrm{S}_{516} \mathrm{~B}_{140} \mathrm{M}_{118}$ & 516 & 140 & 118 & 0.15 & 0.73 & 0.11 & 73.5 & 1.05 \\
\hline $\mathrm{S}_{516} \mathrm{H}_{140} \mathrm{M}_{118}$ & 516 & 140 & 118 & 0.15 & 0.60 & 0.27 & 90 & 1.05 \\
\hline
\end{tabular}

${ }^{1}$ H-NMR Measurements. Spectra were recorded on a Bruker Ultrashield 300 machine with a $300 \mathrm{MHz}$ operating frequency using deuterated chloroform as solvent.

Size Exclusion Chromatography (SEC). SEC measurements were performed on a set of 30 cm SDV-gel columns of $5 \mathrm{~mm}$ particle size having a pore size of $10^{5}, 10^{4}, 10^{3}$, and $10^{2} \AA$ with refractive index and UV $(\lambda=254 \mathrm{~nm})$ detection. SEC was measured at an elution rate of $1 \mathrm{~mL} / \mathrm{min}$ with THF as eluent and with PS or PMMA as standards.

Transmission Electron Microscopy (TEM). Bright field transmission electron microscopy with an acceleration voltage of $80 \mathrm{kV}$ was carried out using a Zeiss CEM 902 electron microscope. Samples were prepared by placing one drop of the polymer solution $(0.1 \mathrm{~g} / \mathrm{L})$ onto carbon-coated copper grids. Excess solvent was instantly absorbed by a filter paper. To enhance the contrast of $\mathrm{PB}$ phase, the samples were stained with $\mathrm{OsO}_{4}$ for $3 \mathrm{~h}$ at ambient conditions. 


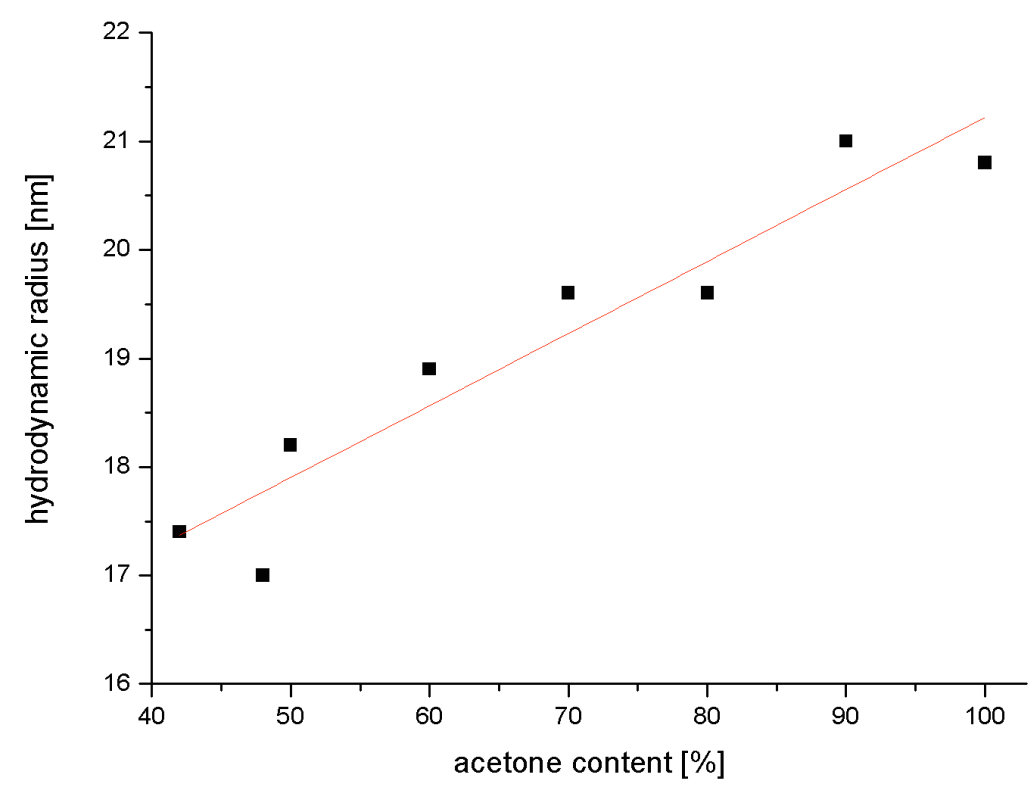

Figure S1: Dependence of hydrodynamic radius of $\mathrm{PMMA}_{5700}$ homopolymer (PDI $=1.20$ ) on the acetone content in acetone/isopropanol mixtures. The polymer is insoluble at acetone contents below 42 vol.- $\%$.

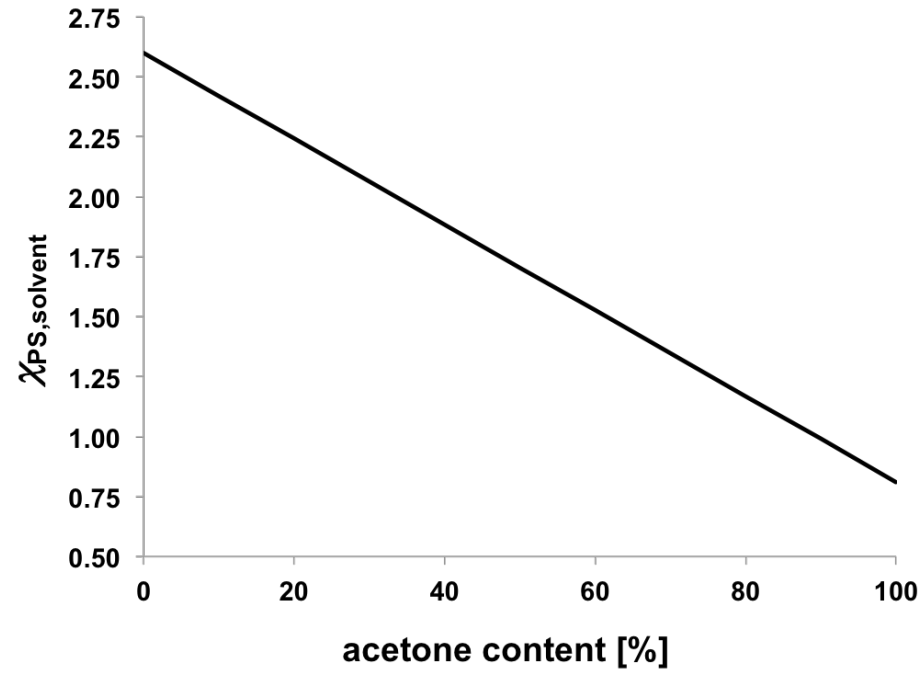

Figure S2: Change of $\chi_{\mathrm{PS}, \text { solvent }}$ in acetone/isopropanol mixtures.

Calculated by linear interpolation of values for $\chi_{\mathrm{PS}, \text { acetone }}=0.81$ and $\chi_{\mathrm{PS}, \mathrm{PPA}}=2$. . $^{3}$ 


\section{Onset of sheet formation}

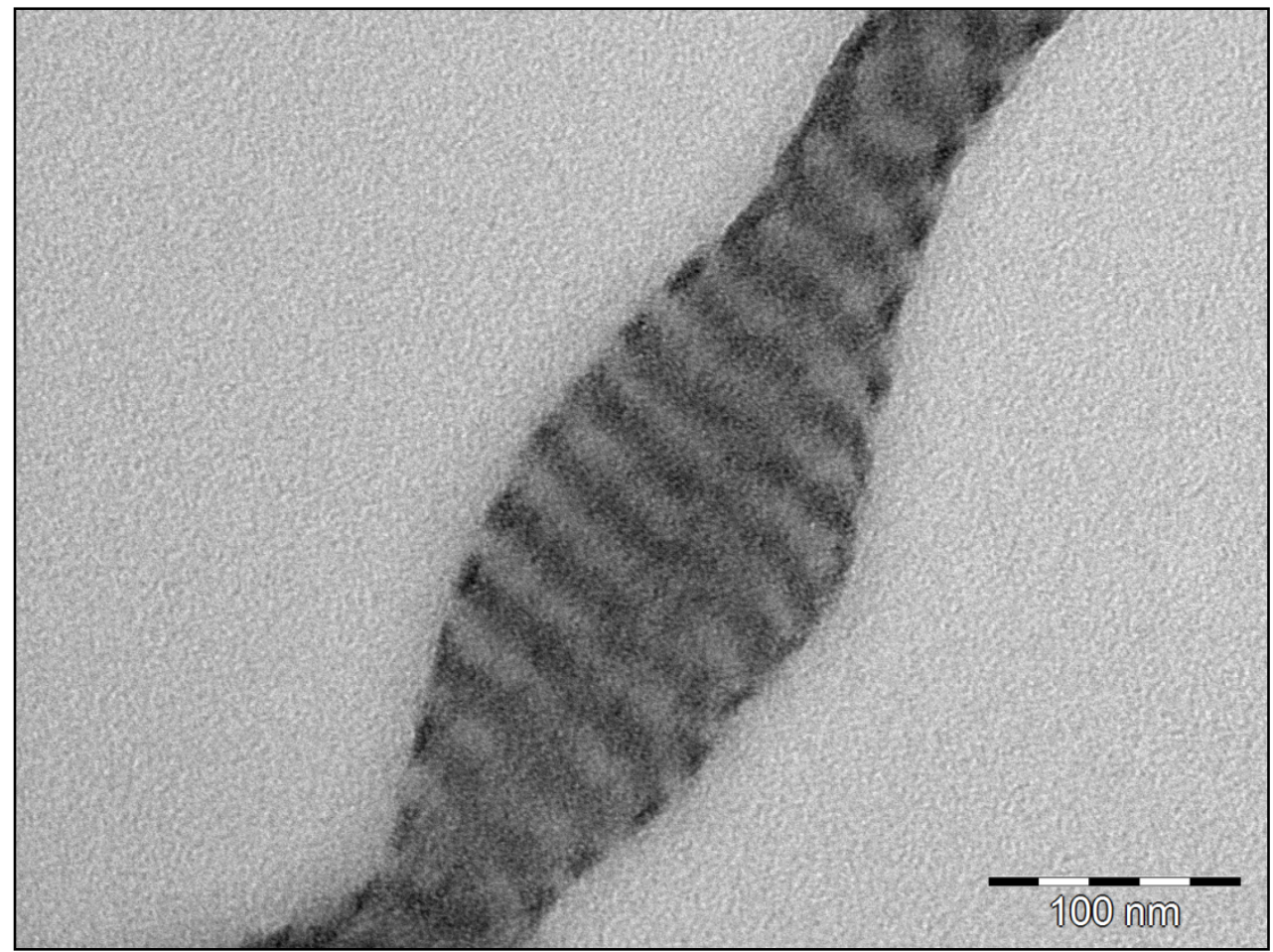

Figure S3: Onset of sheet-formation for $\mathrm{S}_{510} \mathrm{~B}_{258} \mathrm{M}_{260}$ with $x_{\mathrm{PMMA}}=0.25$ in acetone/isopropanol 40:60 (v/v). The transformation of cylindrical micelles to sheets is only observed in some occasions, where the cylinders start to flatten to adopt a sheet-like structure. 


\section{"wet brush" regime "dry brush" regime}

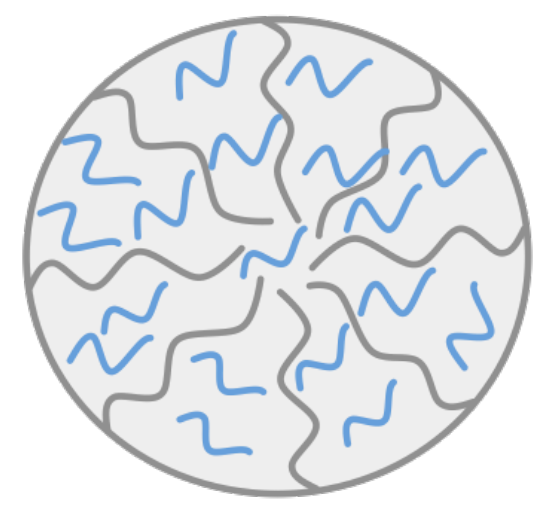

Block Copolymer Chains

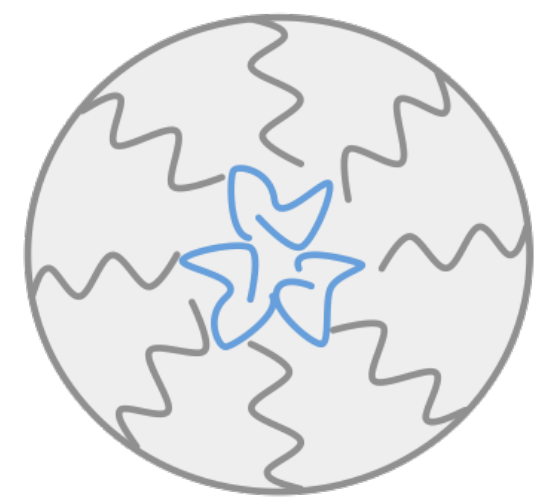

Homopolymer

Chains

Figure S4: Schematic description of the "wet brush" and "dry brush" regime in the core of block copolymer micelles. Short homopolymer chains can interpenetrate the block copolymer chains and homogeneous distribute in the phase ("wet brush" regime). Long homopolymer chains accumulate in the middle of the micellar core since they cannot penetrate the block copolymer brush ("dry brush" regime).

Table S2: Calculation of the apparent change of the molar fraction, $x_{\mathrm{M}}$, of PMMA, the weight fractions $f_{\mathrm{PS}}$ and $f_{\mathrm{PB}}$ of $\mathrm{PS}$ and $\mathrm{PB}$ in the SBM triblock terpolymer and the total molecular weight upon blending with $\mathrm{PS}_{192}$ homopolymer.

\begin{tabular}{ccccc}
\hline $\mathbf{S}_{\mathbf{x}+\mathbf{x}} \mathbf{B}_{\mathbf{y}} \mathbf{M}_{\mathbf{z}}{ }^{\mathbf{M n} a}$ & $\boldsymbol{x}_{\mathrm{PMMA}}{ }^{\boldsymbol{b}}$ & $\boldsymbol{f}_{\mathrm{PS}}{ }^{\boldsymbol{b}}$ & $\boldsymbol{f}_{\mathrm{PB}}{ }^{\boldsymbol{b}}$ & $\begin{array}{c}\boldsymbol{M}_{\mathbf{n}}{ }^{\boldsymbol{1}} \\
{[\mathbf{k g} / \mathbf{m o l}]}\end{array}$ \\
\hline $\mathbf{S}_{\mathbf{5 7 + 0}} \mathbf{B}_{\mathbf{1 5}} \mathbf{M}_{\mathbf{2 8}}{ }^{\mathbf{9 3}}$ & 0.25 & 0.57 & 0.15 & 93 \\
$\mathbf{S}_{\mathbf{5 7 + 4}} \mathbf{B}_{\mathbf{1 4}} \mathbf{M}_{\mathbf{2 5}}{ }^{103}$ & 0.23 & 0.61 & 0.14 & 103 \\
$\mathbf{S}_{\mathbf{5 7 + 8}} \mathbf{B}_{\mathbf{1 2}} \mathbf{M}_{\mathbf{2 3}}{ }^{113}$ & 0.21 & 0.65 & 0.12 & 113 \\
$\mathbf{S}_{\mathbf{5 7 + 1 3}} \mathbf{B}_{\mathbf{1 0}} \mathbf{M}_{\mathbf{2 0}}{ }^{173}$ & 0.14 & 0.70 & 0.10 & 173
\end{tabular}

${ }^{a} \mathrm{x}, \mathrm{y}, \mathrm{z}$ denote the weight fraction of the blocks in \%. The superscript shows the apparent molecular weight in $\mathrm{kg} / \mathrm{mol}$.

${ }^{b}$ Apparent change of the molar fraction of the PMMA block, $x_{\mathrm{M}}$ the weight fractions, $f_{\mathrm{PS}}$ and $f_{\mathrm{PB}}$ of PS and PB and the overall molecular weight $\mathrm{Mn}$. 


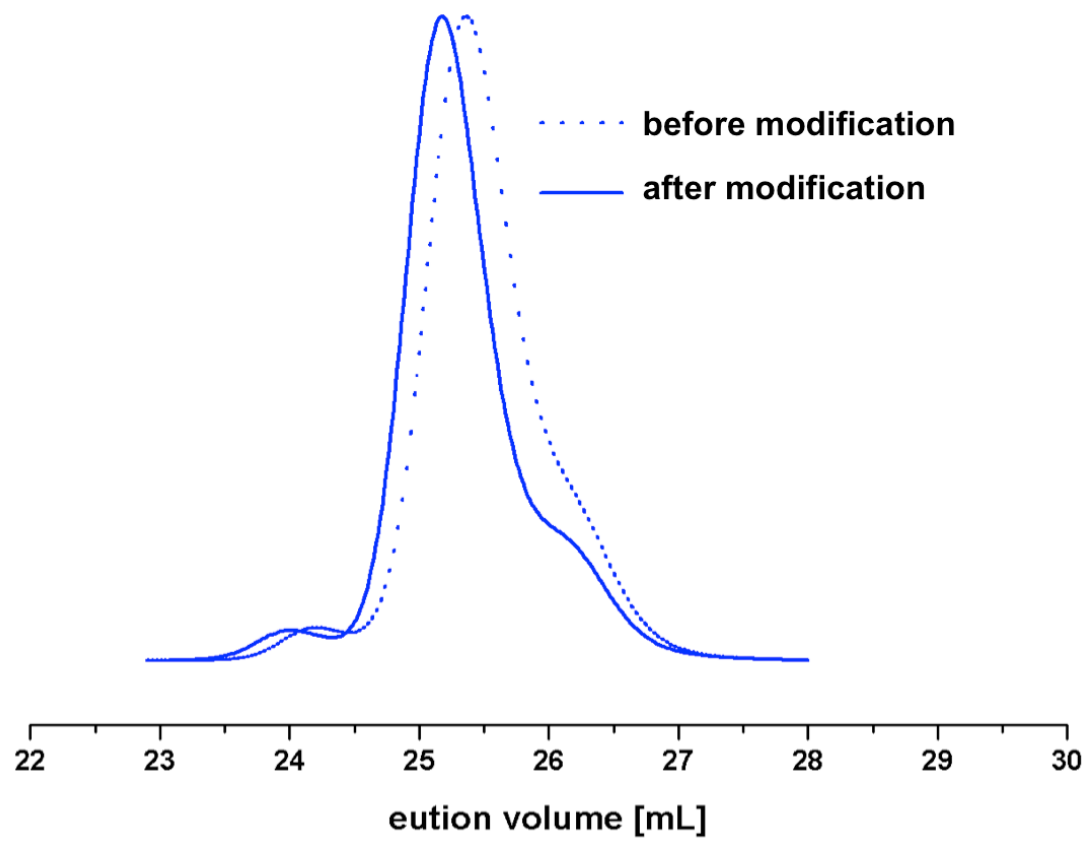

Figure S5: SEC traces of $\mathrm{S}_{306} \mathrm{~B}_{151} \mathrm{M}_{340}$ before (dotted) and after (solid line) thiol-ene click reaction.
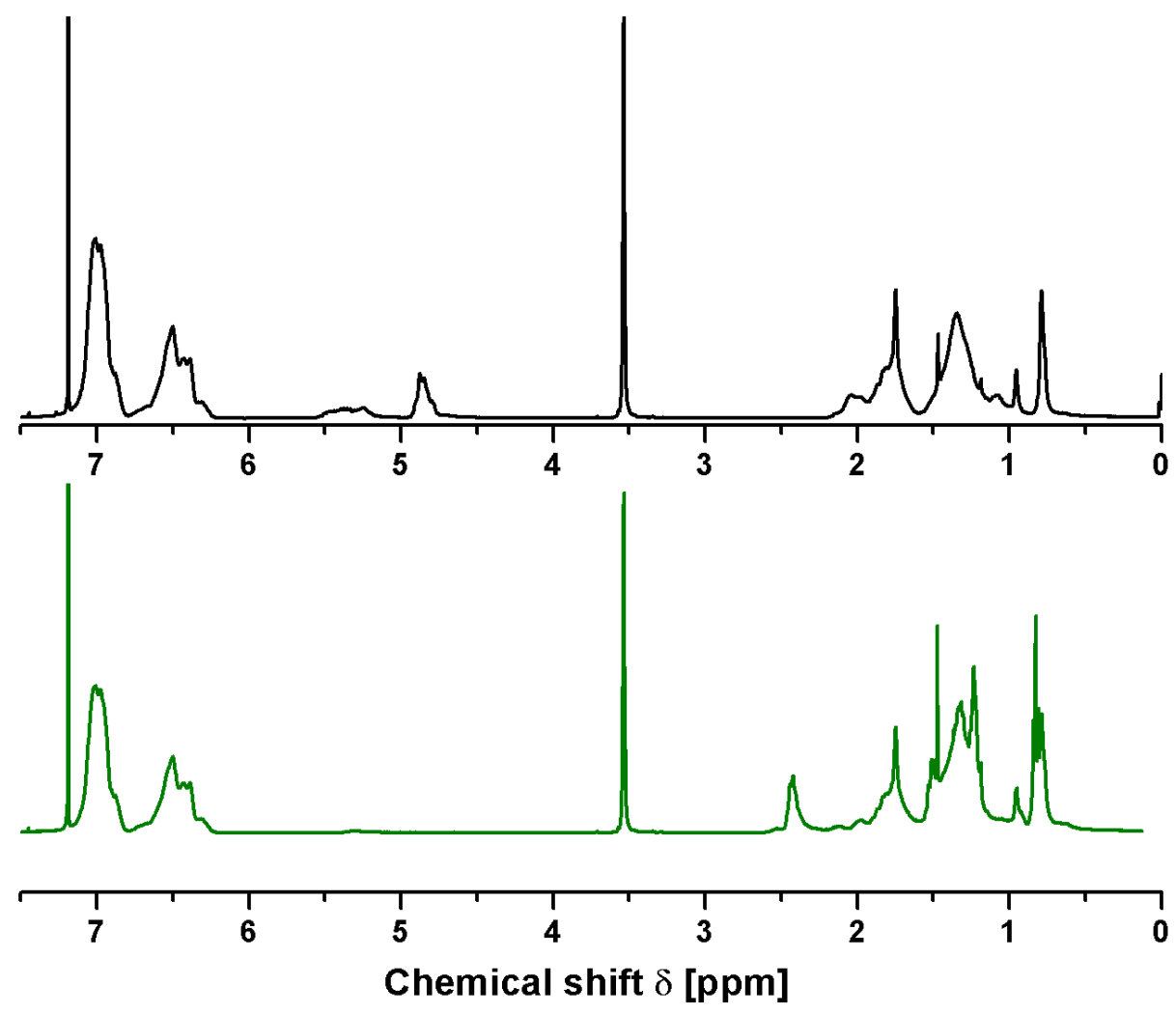

Figure S6: ${ }^{1} \mathrm{H}-\mathrm{NMR}$ spectra of $\mathrm{S}_{306} \mathrm{~B}_{151} \mathrm{M}_{340}$ before (black) and after (green) thiol-ene click reaction. Vanishing of the 1,2-PB double bond peaks between 4.5-5.5 ppm shows that the click reaction was quantitative. There is a marginal peak between 5.0-5.5 originating from 1,4-PB moieties that are not accessible for click reaction (11 wt.-\% 1,4-PB within the SBM triblock terpolymer). 


\section{Supporting References}

(1) Auschra, C.; Stadler, R. Polym. Bull. 1993, 30, 257-264.

(2) Ruckdäschel, H.; Sandler, J. K. W.; Altstädt, V.; Rettig, C.; Schmalz, H.; Abetz, V.; Müller, A. H. E. Polymer 2006, 47, 2772-2790.

(3) Orwall, R.A., Arnold, P.A. in Physical Properties of Polymers Handbook, $2^{\text {nd }}$ ed.; Mark, J., Eds.; Springer Science and Business Media: New York, 2007; p 233-258 